J. Korean Math. Soc. 50 (2013), No. 5, pp. 973-989

http://dx.doi.org/10.4134/JKMS.2013.50.5.973

\title{
ARMENDARIZ PROPERTY OVER PRIME RADICALS
}

\author{
Juncheol Han, Hong Kee Kim, and Yang Lee
}

\begin{abstract}
We observe from known results that the set of nilpotent elements in Armendariz rings has an important role. The upper nilradical coincides with the prime radical in Armendariz rings. So it can be shown that the factor ring of an Armendariz ring over its prime radical is also Armendariz, with the help of Antoine's results for nil-Armendariz rings. We study the structure of rings with such property in Armendariz rings and introduce $A P R$ as a generalization. It is shown that APR is placed between Armendariz and nil-Armendariz. It is shown that an APR ring which is not Armendariz, can always be constructed from any Armendariz ring. It is also proved that a ring $R$ is APR if and only if so is $R[x]$, and that $N(R[x])=N(R)[x]$ when $R$ is APR, where $R[x]$ is the polynomial ring with an indeterminate $x$ over $R$ and $N(-)$ denotes the set of all nilpotent elements. Several kinds of APR rings are found or constructed in the precess related to ordinary ring constructions.
\end{abstract}

\section{Introduction}

Throughout this note every ring is associative with identity unless otherwise stated. But every definition shown in this note can be applicable to rings possibly without identity. Given a ring $R$ (possibly without identity), $N_{*}(R), N^{*}(R)$, and $N(R)$ denote the prime radical, the upper nilradical (i.e., sum of nil ideals), and the set of all nilpotent elements in $R$, respectively. It is well-known that $N_{*}(R) \subseteq N^{*}(R) \subseteq N(R)$. We use $R[x]$ to denote the polynomial ring with an indeterminate $x$ over $R$. For $f(x) \in R[x]$, let $C_{f(x)}$ denote the set of all coefficients of $f(x)$. We use $\operatorname{deg} f(x)$ to denote the degree of $f(x)$. Denote the $n$ by $n$ full (resp., upper triangular) matrix ring over $R$ by $\operatorname{Mat}_{n}(R)$ (resp., $U_{n}(R)$ ). Let $D_{n}(R)$ denote the subring $\left\{m \in U_{n}(R) \mid\right.$ the diagonal entries of $m$ are all equal $\}$ of $U_{n}(R)$. Use $e_{i j}$ for the matrix with $(i, j)$-entry 1 and elsewhere $0 . \mathbb{Z}_{n}$ denotes the ring of integers modulo $n$.

Received July 10, 2012

2010 Mathematics Subject Classification. 16N40, 16S36.

Key words and phrases. APR ring, prime radical, upper nilradical, nil-Armendariz ring, Armendariz ring, polynomial ring.

This research was supported by Basic Science Research Program through the National Research Foundation of Korea(NRF) funded by the Ministry of Education(201306530001). 
A ring is called reduced if it has no nonzero nilpotent elements. For a reduced ring $R$, Armendariz [4, Lemma 1] proved that

$$
a b=0 \text { for all } a \in C_{f(x)}, b \in C_{g(x)} \text { whenever } f(x) g(x)=0
$$

where $f(x), g(x) \in R[x]$. Based on this result, Rege et al. [29] called a ring (possibly without identity) Armendariz if it satisfies this property. So reduced rings are clearly Armendariz. This fact will be used freely in this note. A ring is called Abelian if every idempotent is central. Armendariz rings are Abelian by the proof of [1, Theorem 6] or [18, Corollary 8].

Antoine [2] called a ring $R$ nil-Armendariz if $a b \in N(R)$ for all $a \in C_{f(x)}$ and $b \in C_{g(x)}$ whenever $f(x) g(x) \in N(R)[x]$ for $f(x), g(x) \in R[x]$. Antoine showed that Armendariz rings are nil-Armendariz in [2, Proposition 2.7]. But the converse need not hold with the help of [2, Proposition 2.8] since Armendariz rings are Abelian. Antoine also showed that if $R$ is a nil-Armendariz ring, then $N(R)$ forms a subring of $R$, in [2, Theorem 3.2]. While, Kim et al. proved that a ring $R$ is nil-Armendariz if and only if $a b \in N^{*}(R)$ for all $a \in C_{f(x)}, b \in$ $C_{g(x)}$ whenever $f(x) g(x) \in N^{*}(R)$ for $f(x), g(x) \in R[x]$ in [22, Theorem 11]. Applying this result onto prime radicals, we will call a ring $R$ Armendariz-overprime-radical (simply, $A P R$ ) provided that

$$
f(x) g(x) \in N_{*}(R)[x] \text { implies } a b \in N_{*}(R) \text { for all } a \in C_{f(x)} \text { and } b \in C_{g(x)},
$$

where $f(x), g(x) \in R[x]$. So $R$ is APR if and only if $R / N_{*}(R)$ is Armendariz. $N_{0}(R)$ means the Wedderburn radical (i.e., the sum of all nilpotent ideals) of given a ring $R$.

Lemma 1.1. (1) [23, Lemma 2.3(5)] If a ring $R$ is an Armendariz ring, then $N_{0}(R)=N_{*}(R)=N^{*}(R)$.

(2) [2, Proposition 2.7] Armendariz rings are nil-Armendariz.

(3) $\left[2\right.$, Theorem 3.5] A ring $R$ is nil-Armendariz if and only if $R / N^{*}(R)$ is Armendariz.

(4) The class of Armendariz rings is closed under subrings (possibly without identity) and direct products.

(5) If $R$ is an Abelian ring, then $R / N$ is also Abelian for every nonzero nil ideal $N$ of $R$.

(6) Let $R$ be an $A P R$ ring. If $f_{1}, \ldots, f_{n} \in R[x]$ are such that $f_{1} \cdots f_{n} \in$ $N_{*}(R)[x]$, then $a_{1} \cdots a_{n} \in N_{*}(R)$ for all $a_{i} \in C_{f_{i}}$.

(7) If $R$ is an $A P R$ ring, then $N_{*}(R)=N^{*}(R)$.

(8) If a nil ring is $A P R$, then it is a prime radical ring.

Proof. (4) is easily proved, and (5) is shown by [27, Proposition 3.7.2].

(6) The proof is quite similar to one of $[1$, Proposition 1], but we write it for completeness. Let $f_{1} \cdots f_{n} \in N_{*}(R)[x]$ for $f_{1}, \ldots, f_{n} \in R[x]$. Then $f_{1}\left(f_{2} \cdots f_{n}\right) \in N_{*}(R)[x]$, so $a_{1} b \in N_{*}(R)$ for any $a_{1} \in C_{f_{1}}$ and $b \in C_{f_{2} \cdots f_{n}}$ since $R$ is APR. This yields $a_{1}\left(f_{2} \cdots f_{n}\right) \in N_{*}(R)[x]$ and $\left(a_{1} f_{2}\right)\left(f_{3} \cdots f_{n}\right) \in$ $N_{*}(R)[x]$. Since $R$ is APR and $a_{1} a_{2} \in C_{a_{1} f_{2}}$ for $a_{2} \in C_{f_{2}}$, we have $\left(a_{1} a_{2}\right) c \in$ 
$N_{*}(R)$ for $c \in C_{f_{3} \cdots f_{n}}$. This yields $a_{1} a_{2}\left(f_{3} \cdots f_{n}\right) \in N_{*}(R)[x]$. Continuing, we finally obtain that $a_{1} \cdots a_{n} \in N_{*}(R)$ for all $a_{i} \in C_{f_{i}}$.

(7) Let $R$ be an APR ring. Then $\bar{R}=R / N_{*}(R)$ is Armendariz by definition, and so $N^{*}(\bar{R})=N_{*}(\bar{R})$ by $(1)$. But $N^{*}(R) / N_{*}(R)=N_{*}(\bar{R})=N_{*}\left(R / N_{*}(R)\right)=$ 0 , entailing $N_{*}(R)=N^{*}(R)$.

(8) is an immediate consequence of $(7)$.

Comparing Lemma 1.1(1) and (7), one may conjecture that if $R$ is an APR ring, then $N_{0}(R)=N_{*}(R)=N^{*}(R)$. However there exists a counterexample by [25, Example 2.4(2)].

Example 1.2. Let $K$ be a field and $K\langle x, y\rangle$ be the free algebra generated by noncommuting indeterminates $x, y$ over $K$. Following [25, Example 2.4(2)], consider an infinite word $w=$ yxyxxyxxyxxxyxxxx $\cdots=\prod_{i=1}^{\infty} y x^{i}$, let $I$ be the ideal of $K\langle x, y\rangle$ generated by the set of all words each of which is not a subword of $w$, and $R=K\langle x, y\rangle / I$. Then $N_{*}(R)=R \bar{y} R=N^{*}(R)=$ $N(R)$, whence $R$ is $\mathrm{APR}$ since $R / N_{*}(R)$ is reduced. But $\bar{y} \notin N_{0}(R)$ by the computation in [25, Example 2.4(2)].

Recall that Armendariz rings are Abelian, so we get the following by Lemma 1.1(2), (5) and [2, Proposition 2.4].

Corollary 1.3. Let $R$ be an Armendariz ring. Then $R / N$ is Abelian and nil-Armendariz for any nil ideal $N$ of $R$.

The converse of Lemma 1.1(5) need not hold as we see in $R=U_{2}(A)$ over a reduced $\operatorname{ring} A$. Note that $N=\left(\begin{array}{cc}0 & A \\ 0 & 0\end{array}\right)$ is the only nonzero nil ideal of $R$. $R / N$ is Abelian but $R$ is non-Abelian. If $N$ is non-nil, then Lemma 1.1(5) need not hold. Let $F$ be a field and $R=F\langle a, b\rangle$ be the free algebra generated by noncommuting indeterminates $a, b$ over $F$. Then $R$ is a domain (hence Abelian). Let $N$ be the ideal of $R$ generated by $a^{2}-a$. Then clearly $N$ is non-nil, and $R / N$ is non-Abelian as can be seen by $\bar{a}^{2}=\bar{a}$ and $\bar{a} \bar{b} \neq \bar{b} \bar{a}$.

Theorem 1.4. (1) APR rings are nil-Armendariz.

(2) Armendariz rings are $A P R$.

(3) For a ring $R$ whose prime factor rings are Armendariz, the following conditions are equivalent:

(a) $R$ is $A P R$;

(b) $R / N_{*}(R)$ is a subdirect product of prime Armendariz rings;

(c) $R / N_{*}(R)$ is a subdirect product of Armendariz rings.

Proof. (1) is obtained by Lemma 1.1(3), (7).

(2) Let $R$ be an Armendariz ring. Then $N_{*}(R)=N^{*}(R)$ by Lemma 1.1(1). Let $f(x) g(x) \in N_{*}(R)[x]$ for $f(x), g(x) \in R[x]$. Then we also have $f(x) g(x) \in$ $N^{*}(R)$. But since $R$ is Armendariz, $R$ is nil-Armendariz by Lemma 1.1(2) and so Lemma 1.1(3) implies $a b \in N^{*}(R)=N_{*}(R)$ for all $a \in C_{f(x)}$ and $b \in C_{g(x)}$, entailing $R$ is APR. 
(3) Let $R$ be an APR ring whose prime factor rings are Armendariz. Then $R / N_{*}(R)$ is a subdirect product of prime Armendariz rings. The remainder is obtained from the fact that the class of Armendariz rings is closed under direct products and subrings.

Here one may suspect that prime factor rings of Armendariz rings are also Armendariz. However there exist examples erasing the possibility. Let $R$ be the domain of quaternions with integer coefficients (hence Armendariz). But for any odd prime $p \geq 3$, the ring $R / p R$ is isomorphic to $\operatorname{Mat}_{2}\left(\mathbb{Z}_{p}\right)$ by the argument in $[14$, Exercise $2 \mathrm{~A}]$. Thus $R / p R$ is not Armendariz for any prime $p \geq 3$. $3.2]$.

We obtain the following by Theorem 1.4, Lemma 1.1(3), and [2, Theorem

Corollary 1.5. (1) Let $R$ be a ring. $R$ is Armendariz $\Rightarrow R / N_{*}(R)$ is Armendariz $\Rightarrow R / N^{*}(R)$ is Armendariz.

(2) If $R$ is an APR ring, then $N(R)$ forms a subring of $R$ without identity.

Homomorphic images of Armendariz rings need not be Armendariz as above. But we refer to another example provided by Anderson and Camillo [1, Example 10]. Following to them, let $K$ be a field and $R=K[s, t]$ be the polynomial ring with commuting indeterminates $s, t$ over $K$. Consider the factor ring $S=$ $R /\left(s^{2} R+t^{2} R\right)$. Then $S$ is not Armendariz by the equality $(s+t x)(s-t x)=0$ with $s t \neq 0$.

A ring $R$ is called (von Neumann) regular if for each $a \in R$ there exists $x \in R$ such that $a=a x a$. Von Neumann regular rings are semiprime by [13, Corollary 1.2]; hence a regular ring $R$ is APR if and only if Armendariz if and only if $R$ is Abelian if and only if $R$ is reduced with the help of Corollary 1.5(2) and $[13$, Theorem 3.2].

Marks [28] called a ring $R N I$ if $N^{*}(R)=N(R)$. It is obvious that $R$ is NI if and only if $N(R)$ forms a two-sided ideal if and only if $R / N^{*}(R)$ is reduced. NI rings are nil-Armendariz by [2, Proposition 2.1], but there exist Armendariz (hence nil-Armendariz by Lemma 1.1(2)) rings which are not NI by [2, Example 4.8]. The following shows that the converse of Theorem 1.4(1) need not hold.

Example 1.6. We refer the ring in [19, Example 1.2]. Let $S$ be a reduced ring, $n$ be a positive integer and $R_{n}=U_{2^{n}}(S)$. Each $R_{n}$ is an NI ring by [19, Proposition 4.1(1)]. Define a map $\sigma: R_{n} \rightarrow R_{n+1}$ by $A \mapsto\left(\begin{array}{cc}A & 0 \\ 0 & A\end{array}\right)$, then $R_{n}$ can be considered as a subring of $R_{n+1}$ via $\sigma$ (i.e., $A=\sigma(A)$ for $A \in R_{n}$ ). Notice that $D=\left\{R_{n}, \sigma_{n m}\right\}$, with $\sigma_{n m}=\sigma^{m-n}$ whenever $n \leq m$, is a direct system over $I=\{1,2, \ldots\}$. Set $R=\lim R_{n}$ be the direct limit of $D$. Then $R=\cup_{n=1}^{\infty} R_{n}$, and $R$ is an NI (hence nil-Armendariz) ring by [19, Proposition 1.1], and moreover $N_{*}(R)=0$ by [21, Theorem 2.2(1)]. Thus $R$ is not APR by Lemma $1.1(7)$ since $N^{*}(R)=\left\{m=\left(m_{i j}\right) \in R \mid m_{i i}=0\right.$ for all $\left.i\right\} \neq 0$. In fact, consider polynomials $f(x)=e_{11}+e_{12} x, g(x)=e_{22}-e_{12} x$ over $R$. Then $f(x) g(x)=0$, but $e_{11} e_{12}=e_{12} \neq 0$ and $e_{12}\left(-e_{22}\right)=-e_{12} \neq 0$. 
The converse of Theorem 1.4(2) also need not hold by the following.

Example 1.7. Let $T$ be any commutative ring and $R=U_{2}(T)$. Then $N_{*}(T)=$ $N^{*}(T)=N(T)$ by a well-known fact, and so $N_{*}(R)=\left(\begin{array}{cc}N_{*}(T) & T \\ 0 & N_{*}(T)\end{array}\right)$. Thus $R / N_{*}(R) \cong T / N_{*}(T) \oplus T / N_{*}(T)$ and so $R / N_{*}(R)$ is a reduced (hence Armendariz) ring. This implies that $R$ is APR. But $R$ is not Armendariz since it is non-Abelian.

As we see in Example 1.7, $U_{n}(A)$ is APR when given a $\operatorname{ring} A$ satisfies the property that $A / N_{*}(A)$ is reduced. But $\operatorname{Mat}_{n}(A)$ cannot be APR for $n \geq 2$ over any ring $A$ because $\operatorname{Mat}_{n}(A) / N_{*}\left(\operatorname{Mat}_{n}(A)\right)$ cannot be Abelian.

Birkenmeier et al. [8] used the term 2-primal to define a ring with such property as $A$, i.e., a ring $R$ is 2-primal if and only if $N_{*}(R)=N(R)$ if and only if $R / N_{*}(R)$ is reduced. Thus $U_{n}(A)$ is APR if $A$ is a 2-primal ring for $n=1,2, \ldots$ It is obvious that 2-primal rings are NI, but the converse need not hold by Birkenmeier et al. [9, Example 3.3], Marks [28, Example 2.2], or [19, Example 1.2].

Thinking of $U_{n}(A)$ being APR over a 2-primal ring $A$, one can conclude that APR rings need not be Abelian. This is compared with the fact that Armendariz rings are Abelian. Abelian rings are also need not be APR by the following.

Example 1.8. We refer the ring in [21, Theorem 2.2(2)]. Let $S$ be a reduced ring, $n$ be a positive integer and $R_{n}=D_{2^{n}}(S)$. Each $R_{n}$ is a 2-primal ring by [8, Proposition 2.5]. Define a map $\sigma: R_{n} \rightarrow R_{n+1}$ by $A \mapsto\left(\begin{array}{cc}A & 0 \\ 0 & A\end{array}\right)$, then $R_{n}$ can be considered as a subring of $R_{n+1}$ via $\sigma$ (i.e., $A=\sigma(A)$ for $A \in R_{n}$ ). Notice that $D=\left\{R_{n}, \sigma_{n m}\right\}$, with $\sigma_{n m}=\sigma^{m-n}$ whenever $n \leq m$, is a direct system over $I=\{1,2, \ldots\}$. Set $R=\lim R_{n}$ be the direct limit of $D$. Then $R=\cup_{n=1}^{\infty} R_{n}$, and $R$ is an Abelian ring by $\left[16\right.$, Lemma 2]. However $N_{*}(R)=0$ by [21, Theorem 2.2(2)], so this yields that $R$ is not APR by [24, Example 3]. In fact, let $f(x)=e_{13}+\left(-e_{12}+e_{13}\right) x$ and $g(x)=e_{24}+\left(e_{24}+e_{34}\right) x$ in $R[x]$, then $f(x) g(x)=0$ but $e_{13}\left(e_{24}+e_{34}\right)=e_{14} \neq 0$, entailing that $R / N_{*}(R) \cong R$ is not Armendariz.

Recall that a regular ring $R$ is APR if and only if Armendariz if and only if $R$ is Abelian if and only if $R$ is reduced. A ring $R$ is usually called $\pi$-regular if for each $a \in R$ there exist a positive integer $n$ and $b \in R$ such that $a^{n}=a^{n} b a^{n}$. Regular rings are clearly $\pi$-regular. So one may ask whether Abelian $\pi$-regular rings are APR or not. However the answer is negative by the following. Let $S$ be a division ring in Example 1.8. Then $R$ in Example 1.8 is an Abelian $\pi$-regular ring by the argument in [20, Example 1.5]. But $R$ is not APR by Example 1.8.

Recall that the class of Armendariz rings is closed under subrings. So one may naturally ask whether subrings of APR rings are APR. However, in fact, we do not know of any example of an APR ring that has a non-APR subring. We raise this argument as a question. 
Question. Is the class of APR rings closed under subrings?

But we next examine some kinds of subrings that inherit the APR property. Let $R$ be a ring. Following [27], $a \in R$ is strongly nilpotent if any sequence $a_{0}=$ $a ; a_{1} \in a_{0} R a_{0} ; a_{2} \in a_{1} R a_{1} ; \cdots ; a_{n+1} \in a_{n} R a_{n} ; \cdots$ becomes zero ultimately. By [27, Proposition 3.2.1], $N_{*}(R)$ is the set of all strongly nilpotent elements of $R$.

Proposition 1.9. Let $R$ be an APR ring and $S$ be a subring (possibly without identity).

(1) If $N_{*}(S) \subseteq N_{*}(R)$, then $S$ is $A P R$.

(2) If $S$ is a proper ideal of $R$, then $S$ is $A P R$ as a ring without identity.

(3) If $R$ is an NI ring, then $S$ is $A P R$.

(4) If $e^{2}=e \in R$ is central in $R$, then $e R$ is $A P R$.

Proof. (1) Assume $N_{*}(S) \subseteq N_{*}(R)$. Let $f(x) g(x) \in N_{*}(S)[x]$ for $f(x), g(x) \in$ $S[x]$. Then $f(x) g(x) \in N_{*}(R)[x]$ by assumption. Since $R$ is APR, $a b \in N_{*}(R)$ for all $a \in C_{f(x)}$ and $b \in C_{g(x)}$. The inclusion $S \cap N_{*}(R) \subseteq N_{*}(S)$ is naturally obtained from the elementary fact that the prime radical is the set of all strongly nilpotent elements. This yields $S \cap N_{*}(R)=N_{*}(S)$, combining the assumption $N_{*}(S) \subseteq N_{*}(R)$. So we have $a b \in N_{*}(S)$. Thus $S$ is APR.

(2) If $S$ is an ideal of $R$, then $N_{*}(S) \subseteq N_{*}(R)$ through a simple computation, so $S$ is APR by (1).

(3) Let $R$ be NI. Then $N^{*}(R)=N(R)$. But since $R$ is $\mathrm{APR}, N_{*}(R)=N^{*}(R)$ by Lemma 1.1(7), entailing $N_{*}(R)=N^{*}(R)=N(R)$. So $R$ is 2-primal, and this yields that $S$ is 2-primal (hence APR) by [8, Proposition 2.2]. But we here take another proof, using only a result in this note.

Since $R$ is APR, $N(R)[x]=N(R[x])$ by Theorem $2.1(2)$ to follow, so this yields $N(S)[x] \subseteq S[x] \cap N(R[x])=N(S[x])$. Next let $f(x) g(x) \in N_{*}(S)[x]$ for $f(x), g(x) \in S[x]$. Then

$$
f(x) g(x) \in N_{*}(S)[x] \subseteq N(S)[x] \subseteq N(S[x]) \subseteq N(R[x])=N(R)[x]=N_{*}(R)[x]
$$

by the result above. But since $R$ is APR, we get $a b \in N_{*}(R)$ for all $a \in C_{f(x)}$ and $b \in C_{g(x)}$. This yields $a b \in S \cap N_{*}(R) \subseteq N_{*}(S)$, concluding that $S$ is APR.

(4) Assume that $e^{2}=e \in R$ is central in $R$. Note $e R \cap N_{*}(R)=$ $e N_{*}(R)$. Given $e b \in e R$, consider a sequence $e b_{0}, e b_{1}, e b_{2}, \ldots, e b_{n}, \ldots$ with $e b_{0}=e b, e b_{1} \in e b_{0} R e b_{0}=e b_{0} e R e b_{0}, e b_{2} \in e b_{1} R e b_{1}=e b_{1} e R e b_{1}, \ldots, e b_{n} \in$ $e b_{n-1} R e b_{n-1}=e b_{n-1} e R e b_{n-1}, \ldots$ Then this is eventually zero if $e b \in N_{*}(e R)$. Thus $e b \in N_{*}(R)$, so $e b \in e R \cap N_{*}(R)=e N_{*}(R)$, entailing $N_{*}(e R) \subseteq e N_{*}(R)$. Consequently $N_{*}(e R)=e N_{*}(R)$, and thus $e R$ is APR by (1).

We denote the direct product (resp. direct sum) by $\prod$ (resp. $\oplus$ ).

Corollary 1.10. Let $R_{i}$ be rings for $i \in I$. Then $\prod_{i \in I} R_{i}\left(\oplus_{i \in I} R_{i}\right)$ is APR if and only if $R_{i}$ is $A P R$ for all $i \in I$, where $\oplus_{i \in I} R_{i}$ is a ring possibly without identity. 
Proof. It is simply shown that $N_{*}\left(\prod_{i \in I} R_{i}\right)=\prod_{i \in I} N_{*}\left(R_{i}\right)$ and $N_{*}\left(\oplus_{i \in I} R_{i}\right)=$ $\oplus_{i \in I} N_{*}\left(R_{i}\right)$, entailing $\frac{\prod_{i \in I} R_{i}}{N_{*}\left(\prod_{i \in I} R_{i}\right)} \cong \prod_{i \in I} \frac{R_{i}}{N_{*}\left(R_{i}\right)}$ and $\frac{\oplus_{i \in I} R_{i}}{N_{*}\left(\oplus_{i \in I} R_{i}\right)} \cong \oplus_{i \in I} \frac{R_{i}}{N_{*}\left(R_{i}\right)}$. So the proof is obtained by Proposition 1.9(4) and the fact that $\left(\oplus_{i \in I} R_{i}\right)[x] \cong$ $\oplus_{i \in I} R_{i}[x]$ and $\left(\prod_{i \in I} R_{i}\right)[x]$ is isomorphic to a subring of $\prod_{i \in I} R_{i}[x]$.

As a special case of Corollary 1.10, we have that a ring $R$ is APR if and only if $e R$ and $(1-e) R$ are both APR for a central idempotent $e$ of $R$.

We next find a kind of shape of an ideal $I$ of a ring $R$ so that $R / I$ can be APR. The following is comparable with [2, Proposition 2.4].

Proposition 1.11. Let $R$ be a ring and $I$ be an ideal of $R$ with $I \subseteq N_{*}(R)$. Then $R$ is $A P R$ if and only if so is $R / I$.

Proof. Let $\bar{R}=R / I$. Note $\frac{N_{*}(R)}{I}=N_{*}(\bar{R})$. Suppose that $f(x), g(x) \in R[x]$ satisfy $f(x) g(x) \in N_{*}(R)[x]$. Then $\bar{f}(x) \bar{g}(x) \in N_{*}(\bar{R})[x]$. Since $R / I$ is APR, it follows that $\bar{a} \bar{b} \in N_{*}(\bar{R})$ for all $a \in C_{f(x)}, b \in C_{g(x)}$. This yields $a b \in N_{*}(R)$. The proof of the converse is similar.

Proposition 1.11 need not hold when $I \nsubseteq N_{*}(R)$ as we see in the following. Let $K$ be a field and $R_{1}, R_{2}$ be $K$-algebras. $R_{1} *_{K} R_{2}$ denotes the ring coproduct of $R_{1}$ and $R_{2}$ (see Antoine [2] and Bergman [6,7] for details).

Example 1.12. (1) The first construction is essentially due to [2, Theorem 4.7]. Let $K$ be a field and $A$ be a $K$-algebra. Let $C=K[b]$ be the polynomial ring with an indeterminate $b$ over $K$, and $I$ be the ideal of $C$ generated by $b^{n}$ for $n \geq 2$. Set $B=C / I$ and $R=A *_{K} B$. Then the following conditions are equivalent by [2, Theorem 4.7], [22, Theorem 1.1], and Theorem 1.4:

(1) $R$ is Armendariz;

(2) $R$ is APR;

(3) $R$ is nil-Armendariz;

(4) $A$ is a domain and $U(A)=K \backslash\{0\}$;

(5) $N(R)$ forms a subring of $R$, where $U(A)$ means the group of units in $A$.

Next we use the ring in [22, Example 1.2(2)]. Let $K$ be the field of integers modulo 2 and $C=K[a]$ the polynomial ring with an indeterminate $a$ over $K$. Let $D=K[b]$ be the polynomial ring with an indeterminate $b$ over $K$. Let $J$ be the ideal of $D$ generated by $b^{2}$. Set $A=C \oplus C$ and $B=D / J$. Identify $b$ with its image for simplicity. Then $b^{2}=0$. Put $R=A *_{K} B$. Then $U(A)=K \backslash\{0\}$ but $A$ is not a domain; hence $R$ is not APR by the preceding result. In fact, letting

$$
f(x)=b(a, 0)+b(a, 0) b x \text { and } g(x)=(0, a)-b(0, a) x \in R[x],
$$

$f(x) g(x)=0$ but $b(a, 0) b(0, a)$ is non-nilpotent (i.e., $\left.b(a, 0) b(0, a) \notin N_{*}(R)\right)$. Note that $A *_{K} D$ is reduced and $R=\frac{A *_{K} D}{I}, I \neq 0$, and $N_{*}\left(A *_{K} D\right)=0$ where $I$ is the ideal of $A *_{K} D$ generated by $J$. 
(2) Let $A$ be a semiprime APR ring and $R=A \oplus \operatorname{Mat}_{n}(A)$ with $n \geq 2$. Then $N_{*}(R)=0$. Letting $I=0 \oplus \operatorname{Mat}_{n}(A)$, we have an APR ring $\frac{R}{I} \cong A$. But $R$ is not APR by Corollary 1.10. Note $I \nsubseteq N_{*}(R)$.

Homomorphic images of APR (even if reduced) rings need not be APR by Example 1.12(1).

In the following we find a criterion by which we can find APR homomorphic images. Huh et al. showed in [18, Theorem 11] that if $R / I$ is an Armendariz ring and $I$ is a reduced ring (without identity), then $R$ is Armendariz, where $R$ is a ring and $I$ is a proper ideal of $R$. In this situation, $R$ need not be Armendariz when $I$ is Armendariz but not reduced, as can be seen by $U_{2}(A)$ over a reduced ring $A$. But $R$ will be shown to be APR in such a case as in the preceding argument.

Theorem 1.13. Let $R$ be a ring and $I$ be a proper ideal of $R$ with $N_{*}(R) \subseteq I$. If $R / I$ is an Armendariz ring and $I$ is a 2-primal ring (without identity), then $R$ is $A P R$.

Proof. Suppose that $\bar{R}=R / I$ is Armendariz and $I$ is 2-primal. From $N_{*}(R) \subseteq$ $I$, we have $N_{*}(R) \subseteq N_{*}(I)$. Since $I$ is an ideal of $R, N_{*}(I) \subseteq N_{*}(R)$ and so $N_{*}(R)=N_{*}(I)$. Since $I$ is 2-primal, $I / N_{*}(R)$ is reduced.

Let $f(x) g(x) \in N_{*}(R)[x]$ for $f(x)=\sum_{i=0}^{m} a_{i} x^{i}, g(x)=\sum_{j=0}^{n} b_{j} x^{j} \in R[x]$. Then $N_{*}(R) \subseteq I$ implies $f(x) g(x) \in I[x]$, and since $R / I$ is Armendariz, $a_{i} b_{j} \in I$ for all $i, j$.

We apply the proof of [18, Theorem 11], and proceed by induction on $m$. If $m=0$, then we are done, so suppose $m \geq 1$. From $f(x) g(x) \in N_{*}(R)[x]$, $a_{0} b_{0} \in N_{*}(R)$.

Here assume that $a_{0} b_{l} \notin N_{*}(R)$ for some $l$. Take $s \in\{1,2, \ldots, n\}$ such that $s$ is the smallest one with respect to the property $a_{0} b_{s} \notin N_{*}(R)$. So $a_{0} b_{k} \in N_{*}(R)$ for all $k \in\{0, \ldots, s-1\}$. Now consider a product

$$
\left(a_{s-k} b_{k}\right)\left(a_{0} b_{s}\right)^{2}=a_{s-k} b_{k}\left(a_{0} b_{s}\right) a_{0} b_{s} .
$$

Letting $c=b_{k}\left(a_{0} b_{s}\right) a_{0}, c \in I$ and $c^{2}=b_{k}\left(a_{0} b_{s}\right)\left(a_{0} b_{k}\right)\left(a_{0} b_{s}\right) a_{0} \in N_{*}(R)$, so $c \in N_{*}(R)$ (hence $\left.\left(a_{s-k} b_{k}\right)\left(a_{0} b_{s}\right)^{2} \in N_{*}(R)\right)$ since $I / N_{*}(R)$ is reduced.

The coefficient of the term of degree $s$ in $f(x) g(x) \in N_{*}(R)[x]$ is $a_{0} b_{s}+$ $a_{1} b_{s-1}+\cdots+a_{s} b_{0}=a_{0} b_{s}+\sum_{k=0}^{s-1} a_{s-k} b_{k} \in N_{*}(R)$ (letting $a_{v}=0, b_{w}=0$ for some $v, w$ if necessary). Multiplying $\left(a_{0} b_{s}\right)^{2}$ to this sum on the right side, we obtain

$$
\left(a_{0} b_{s}+\sum_{k=0}^{s-1} a_{s-k} b_{k}\right)\left(a_{0} b_{s}\right)^{2}=\left(a_{0} b_{s}\right)^{3}+p \in N_{*}(R)
$$

for some $p \in N_{*}(R)$ by the preceding result. Then $\left(a_{0} b_{s}\right)^{3} \in N_{*}(R)$, and this yields $a_{0} b_{s} \in N_{*}(R)$ since $I / N_{*}(R)$ is reduced. This induces a contradiction, and hence $a_{0} b_{j} \in N_{*}(R)$ for all $j$. Thus we have $a_{0} g(x) \in N_{*}(R)[x]$, so $\left(a_{1} x+\right.$ $\left.\cdots+a_{m} x^{m}\right) g(x) \in N_{*}(R)[x]$. This yields $\left(a_{1}+\cdots+a_{m} x^{m-1}\right) g(x) \in N_{*}(R)[x]$. 
Letting $f_{1}(x)=a_{1}+\cdots+a_{m} x^{m-1}, f_{1}(x) g(x) \in N_{*}(R)[x]$ with $\operatorname{deg} f_{1}(x)=$ $m=1$. So, by the induction hypothesis, we get $a_{h} b_{j} \in N_{*}(R)$ for all $h, j$ with $1 \leq h \leq m$. Consequently $a_{i} b_{j} \in N_{*}(R)$ for all $i, j$.

Let $A$ be a 2-primal ring and $R=U_{n}(A)$. Then $R$ is APR by Theorem 2.1 to follow. But we here apply Theorem 1.13 to show $R$ being APR. Let $I=\left\{m=\left(m_{i j}\right) \in R \mid m_{i i} \in N_{*}(A)\right.$ for all $\left.i\right\}$. Then $I=N_{*}(R)$, and $R / I$ is reduced (hence Armendariz) since $A$ is 2-primal. Moreover $I$ is a 2-primal ring since $I=N_{*}(R)$ implies $N_{*}(I)=I$, whence $R$ is APR by Theorem 1.13.

As another application of Theorem 1.13, consider the Armendariz (hence APR) ring in [2, Example 4.10]. Let $K$ be a field and $A=K\langle a, b\rangle$ be the free algebra generated by noncommuting indeterminates $a, b$ over $K$. Let $H$ be the ideal of $A$ generated by $a b$, and write $B=A / H$. Next let $I$ be the ideal of $B$ generated by $\bar{b}$ and write $R=B / I$. Then $R$ is isomorphic to $K\langle a\rangle=K[a]$ which is a domain. Moreover $N(I)=R \bar{b} \bar{a} R$ with $(R \bar{b} \bar{a} R)^{2}=0$ such that $I / N(I)$ is isomorphic to $K\langle b\rangle=K[b]$ which is a domain. Thus $R$ is APR by Theorem 1.13 .

Any finite dimensional algebra need not be APR as can be seen by $\operatorname{Mat}_{n}(A)$ $(n \geq 2)$ over any (finite) ring $A$. We next investigate some basic form of finite APR rings. We use the term "minimal" to mean "having smallest cardinality". $G F\left(p^{n}\right)$ denotes the Galois field of order $p^{n}$.

Proposition 1.14. (1) Every minimal noncommutative APR ring is isomorphic to $U_{2}\left(\mathbb{Z}_{2}\right)$.

(2) Every minimal non-Armendariz APR ring is isomorphic to $U_{2}\left(\mathbb{Z}_{2}\right)$.

Proof. Eldridge proved that a finite ring is commutative if it has a cube free factorization in [12, Theorem], and that if a ring $A$ is of order $p^{3}, p$ a prime, then $A \cong U_{2}(G F(p))$ in [12, Proposition]. Thus every minimal noncommutative ring is isomorphic to $U_{2}\left(\mathbb{Z}_{2}\right)$. But $U_{2}\left(\mathbb{Z}_{2}\right)$ is 2-primal (hence APR), so this yields that every minimal noncommutative APR ring is isomorphic to $U_{2}\left(\mathbb{Z}_{2}\right)$.

Next assume that the order of a finite ring $R$ is 4 . Then $R$ is commutative by [12, Theorem]. Indeed, we can obtain by applying the proof of [12, Lemma] that $R$ is isomorphic to $G F\left(2^{2}\right)$ or $\mathbb{Z}_{2} \oplus \mathbb{Z}_{2}$ when $R$ is semiprimitive; and $R$ is isomorphic to $\mathbb{Z}_{4}$ or $D_{2}\left(\mathbb{Z}_{2}\right)$ when $R$ is non-semiprimitive. But every case is Armendariz with the help of [24, Proposition 2] and [29, Proposition 2.1].

Therefore we must conclude that every minimal non-Armendariz APR ring is isomorphic to $U_{2}\left(\mathbb{Z}_{2}\right)$.

Proposition 1.15. (1) Let $R$ be a left or right Artinian ring. Then $R$ is APR if and only if $R$ is 2-primal.

(2) Suppose that every prime factor ring of a ring $R$ is left and right Noetherian. Then $R$ is APR if and only if $R$ is 2-primal.

Proof. It suffices to show that if $R$ is APR, then $R$ is 2-primal. 
(1) By hypothesis, $R / N_{*}(R)$ is semisimple Artinian. If $R$ is APR, then $R / N_{*}(R)$ is Armendariz (hence Abelian by [18, Corollary 8]), so this yields that $R / N_{*}(R)$ is a finite direct product of division rings. Thus $R$ is 2-primal since $R / N_{*}(R)$ is reduced.

(2) Let $R$ be APR. Then $R / N_{*}(R)$ is a subdirect product of prime Armendariz rings $R / P_{i}$ by Theorem $1.4(3)$, letting $N_{*}(R)=\cap_{i \in I} P_{i}$. Here every $R / P_{i}$ is reduced (hence a domain) by [1, Theorem 7] since $R / P_{i}$ is left and right Noetherian by hypothesis. This yields that $R / N_{*}(R)$ is reduced, entailing that $R$ is 2-primal.

Every minimal APR ring must be 2-primal by Proposition 1.15, thinking of Proposition 1.14. If given a ring $R$ is left and right Noetherian, then $R$ is APR if and only if $R$ is 2-primal by Proposition 1.15(2).

\section{More examples}

In this section we argue about ordinary ring extensions (e.g., polynomial rings) over APR rings. $R[[x]]$ denotes the power series ring with an indeterminate $x$ over a ring $R$. Let $X$ denote a nonempty set of commuting indeterminates over $R$. The polynomial ring with $X$ over $R$ is denoted by $R[X]$, writing $R[x]$ when $X=\{x\}$.

Theorem 2.1. (1) $A$ ring $R$ is $A P R$ if and only if so is $R[X]$.

(2) If a ring $R$ is $A P R$, then $N(R[X])=N(R)[X]$.

Proof. (1) Let $R$ be an APR ring. Then $R / N_{*}(R)$ is Armendariz, so $\frac{R}{N_{*}(R)}[X] \cong$ $\frac{R[X]}{N_{*}(R)[X]}$ is also Armendariz by [1, Corollary 3]. Note $N_{*}(R)[X]=N_{*}(R[X])$ by $\left[26\right.$, Theorem 10.19], so this yields that $\frac{R[X]}{N_{*}(R)[X]}=\frac{R[X]}{N_{*}(R[X])}$ is Armendariz. This implies that $R[X]$ is APR

Conversely let $R[X]$ be APR. Then $R[X] / N_{*}(R[X])$ is Armendariz, so $\frac{R}{N_{*}(R)}[X]$ is also Armendariz by the argument above. It is obvious that subrings of Armendariz rings are Armendariz. This implies that $R / N_{*}(R)$ is Armendariz, entailing that $R$ is APR.

(2) Let $R$ be an APR ring. Then $R[x]$ is APR by (1). Moreover $R$ and $R[x]$ are both nil-Armendariz by Theorem $1.4(1)$. Thus $N(R[x])=N(R)[x]$ by $[2$, Theorem 5.3]. Consider $R[x, y]$ for $x, y \in X$. By the preceding result, we have

$$
N(R[x, y])=N(R[x])[y]=N(R)[x][y]=N(R)[x, y] .
$$

So we obtain $N\left(R\left[X_{0}\right]\right)=N(R)\left[X_{0}\right]$ inductively for any finite subset $X_{0}$ of $X$. This yields $N(R[X])=N(R)[X]$.

The converse of Theorem 2.1(2) need not hold as can be seen by Example 1.6. Let $R$ be the ring in Example 1.6. Then $R$ is not APR, but $N(R[X])=$ $N(R)[X]$ with $N(R)=\left\{m=\left(m_{i j}\right) \in R \mid m_{i i}=0\right.$ for all $\left.i\right\}$.

Corollary 2.2 ([8, Proposition 2.6]). If $R$ is a 2-primal ring, then so is $R[X]$. 
Proof. Let $R$ be a 2-primal ring. Then $R$ is APR, so $N(R[X])=N(R)[X]$ by Theorem 2.1(2). This yields $N(R[X])=N(R)[X]=N_{*}(R)[X]=N_{*}(R[X])$ by [26, Theorem 10.19].

However Theorem 2.1(1) is not valid for power series rings. To see that, we use the construction and computation in [15, Example 1.1].

Example 2.3. (1) Let $F$ be a field, $V$ be a (left) vector space over $F$ with an infinite basis $\left\{v_{1}, v_{2}, \ldots\right\}$, and $A$ be the endomorphism ring of $V$ over $F$. Following to [15, Example 1.1], let $A_{1}=\left\{f \in A \mid \operatorname{rank}(f)<\infty\right.$ and $f\left(v_{i}\right)=$ $a_{1} v_{1}+\cdots+a_{i} v_{i}$ for $i=1,2, \ldots$ with $\left.a_{j} \in F\right\}$ and, $R$ be the $F$-subalgebra of $A$ generated by $A_{1}$ and $1_{A}$. Then $R$ is 2-primal (hence APR) by the argument in [15, Example 1.1]. Let $f(x)=e_{12} x+\left(e_{34}+e_{56}\right) x^{2}+\cdots+$ $\left(\Sigma_{i=0}^{2^{n-1}-1} e_{\left(2^{n}+2 i-1\right)\left(2^{n}+2 i\right)}\right) x^{n}+\cdots$ and $g(x)=e_{23} x+\left(e_{45}+e_{67}\right) x^{2}+\cdots+$ $\left(\Sigma_{i=0}^{2^{n-1}-1} e_{\left(2^{n}+2 i\right)\left(2^{n}+2 i+1\right)}\right) x^{n}+\cdots$ in $R[[x]]$. Then $f(x)^{2}=0=g(x)^{2}$. If $R[[x]]$ is APR, then $f(x)+g(x)$ is also nilpotent by Theorem 1.4(1) and [2, Theorem $3.2]$, but $f(x)+g(x) \notin N(R[[x]])$ by the computation in [15, Example 1.1], a contradiction. Thus $R[[x]]$ is not APR.

(2) We use [3, Example 1]. Let $K$ be a field and $A=K\langle a, b\rangle$ be the free algebra generated by noncommuting indeterminates $a, b$ over $K$. Let $I$ be the ideal of $A$ generated by $b^{2}$ and $R=A / I$. Then $R[[x]]$ is not nil-Armendariz by [3, Example 1], and so it is not APR by Theorem 1.4(1).

The following is proved by [15, Proposition 1.2], but here we argue about another kind of proof, observing the structure of power series rings concerning the APR property.

Proposition 2.4. If $R$ is a 2-primal ring with nilpotent $N_{*}(R)$, then $R[[x]]$ is $A P R$.

Proof. Let $R$ be a ring. First note that $N_{*}(R[[x]]) \subseteq N_{*}(R)[[x]]$ by [17, Corollary 1.2]. Here if $N_{*}(R)$ is nilpotent, then $N_{*}(R[[x]])=N_{*}(R)[[x]]$. Let $R[[x]][y]$ denote the polynomial ring with an indeterminate $y$ over $R[[x]]$. Then

$$
N_{*}(R[[x]][y])=N_{*}(R[[x]])[y]=N_{*}(R)[[x]][y] \subset N_{*}(R)[y][[x]],
$$

since $A[[x]][y] \subset A[y][[x]]$ for any $\operatorname{ring} A$, where $x, y$ are commuting indeterminates over $A$.

Now let $f(y) g(y) \in N_{*}(R[[x]])[y]$ for $f(y), g(y) \in R[[x]][y]$. Then $f(y) g(y) \in$ $N_{*}(R)[y][[x]]$. We can express $f(y), g(y)$ by

$$
f(y)=\sum_{i=0}^{\infty} s_{i}(y) x^{i}, g(y)=\sum_{j=0}^{\infty} t_{j}(y) x^{j} \text { with } s_{i}(y), t_{j}(y) \in R[y] \text { for all } i, j .
$$

So

$$
f(y) g(y)=\sum_{k=0}^{\infty}\left(\sum_{i+j=k} s_{i}(y) t_{j}(y)\right) x^{k}
$$


If $R$ is a 2-primal ring, then $R / N_{*}(R)$ is reduced and this yields that

$$
\frac{R}{N_{*}(R)}[y][[x]] \cong \frac{R[y]}{N_{*}(R)[y]}[[x]] \cong \frac{R[y][[x]]}{N_{*}(R)[y][[x]]}
$$

is reduced. Thus $s_{i}(y) t_{j}(y) \in N_{*}(R)[y]$ for all $i, j$ by [23, Lemma 2.3](1) since $f(y) g(y) \in N_{*}(R)[y][[x]]$. But $R$ is APR, so $a b \in N_{*}(R)$ for all $a \in C_{s_{i}(y)}$ and $b \in C_{t_{j}(y)}$. This yields that $\alpha \beta \in N_{*}(R)[[x]]=N_{*}(R[[x]])$ for all $\alpha \in C_{f(y)}$ and $\beta \in C_{g(y)}$ since $a$ and $b$ are also coefficients of sum-factors of terms of $f(y)$ and $g(y)$ respectively.

Note that $N_{*}(R)$ in Example 2.3 is not nilpotent.

Based on Theorem 2.1(1), it is also natural to examine the APR property for the cases of skew polynomial rings and differential polynomial rings. But we will also find counterexamples in both cases. Let $R$ be a ring, $\sigma$ be an endomorphism of $R$, and $\delta$ be a $\sigma$-derivation of $R$. In this situation, the Ore extension $R[x ; \sigma, \delta]$ of $R$ usually means the ring obtained by giving $R[x]$ with the new multiplication $x r=\sigma(r) x+\delta(r)$ for $r \in R$. when $\delta=0, R[x ; \sigma, 0]$ is written by $R[x ; \sigma]$ and called an a skew polynomial ring. When $\sigma$ is the identity map, $R[x ; 1, \delta]$ is written by $R[x ; \delta]$ and called a differential polynomial ring.

In the following we see that skew polynomial rings over APR rings need not be APR.

Example 2.5. Let $A$ be a domain and $R=A \oplus A$. Then $R$ is reduced, so APR. Consider the automorphism $\sigma(a, b)=(b, a)$ of $R$ and the skew polynomial ring $R[x ; \sigma]$. Then $R[x ; \sigma]$ is semiprime by the computation in [10, Example 3.1]. Write $S=R[x ; \sigma]$ and let

$$
f(t)=(1,0) x+(1,0) t \text { and } g(t)=(1,0) x^{2}-(0,1) x t \in S[t],
$$

where $S[t]$ is the polynomial ring with an indeterminate $t$ over $S$. Then $f(t) g(t)=0$ but $(1,0) x(0,1) x=(1,0) x^{2} \neq 0$. Since $N_{*}(S)=0, S$ is not APR.

In the following we see that differential polynomial rings over APR rings need not be APR.

Example 2.6. We use the ring and argument in [5, Example 11]. Let $R=$ $\mathbb{Z}_{2}[t] / t^{2} \mathbb{Z}_{2}[t]$ is commutative (hence APR), where $\mathbb{Z}_{2}[t]$ is the polynomial ring with an indeterminate $t$ over $\mathbb{Z}_{2}$. Following [5, Example 11], define a derivation $\delta$ of $R$ by $\delta(t+I)=1+I$, where $I=t^{2} \mathbb{Z}_{2}[t]$. Then $R[x ; \delta] \cong \operatorname{Mat}_{2}\left(\mathbb{Z}_{2}\left[x^{2}\right]\right)$ by the argument in $[5$, Example 11$]$. However $\operatorname{Mat}_{2}\left(\mathbb{Z}_{2}\left[x^{2}\right]\right)$ cannot be APR by the argument after Example 1.7.

Thinking of Theorem 1.4(3) and the fact that subdirect products of Armendariz rings are Armendariz, one may conjecture that subdirect products of APR rings are also APR. We will answer this in the negative. The following is similar to [20, Lemma 2.1]. 
Proposition 2.7. Let $R$ be a ring and $n$ be a positive integer.

(1) $R[x] / x^{n} R[x]$ is APR if and only if so is $R$.

(2) $R[[x]] / x^{n} R[[x]]$ is $A P R$ if and only if so is $R$.

Proof. (1) Let $E=R[x] / x^{n} R[x]$. Then $N_{*}(E)=\frac{N_{*}(R)+x R[x]}{x^{n} R[x]}$ obviously, so $\frac{R}{N_{*}(R)} \cong \frac{E}{N_{*}(E)}$. This completes the proof. The proof of (2) is quite similar to (1).

One may compare Proposition 2.7(1) with [1, Theorem 5]. In the following, we use the constructions in [15, Example 1.1] and [20, Example 2.2].

Example 2.8. Let $R$ be the ring in Example 2.3. Then $R$ is APR but $R[[x]]$ is not APR by the argument in Example 2.3. Following to [20, Example 2.2], define a homomorphism $\sigma: R[[x]] \rightarrow \prod_{n=1}^{\infty} \frac{R[[x]]}{x^{n} R[[x]]}$ with $\sigma(f(x))=(f(x)+$ $\left.x^{n} R[[x]]\right)_{n=1}^{\infty}$. Since the kernel of $\sigma$ is zero, $R[[x]]$ is a subdirect product of $\frac{R[[x]]}{\left.x^{n} R[\mid x]\right]}$ 's. Since $R$ is APR, so is each $\frac{R[[x]]}{\left.x^{n} R[\mid x]\right]}$ by Proposition 2.7(2); hence $R[[x]]$ is a subdirect product of APR rings. However $R[[x]]$ is not APR.

Proposition 2.9. Let $R$ be a ring and $M$ be a multiplicative monoid consisting of central regular elements in $R$. Then $R$ is $A P R$ if and only if so is $M^{-1} R$.

Proof. Let $R$ be APR and $E=M^{-1} R$. Note $N_{*}(E)=M^{-1} N_{*}(R)$. Let $f(x) g(x) \in N_{*}(E)[x]$ for $f(x)=\sum_{i=0}^{m} \alpha_{i} x^{i}, g(x)=\sum_{j=0}^{n} \beta_{j} x^{j} \in E[x]$. Here we can assume that $\alpha_{i}=a_{i} u^{-1}, \beta_{j}=b_{j} v^{-1}$ with $a_{i}, b_{j} \in R$ for all $i, j$ and $u, v \in$ $M$. Then $f(x) g(x)=\sum_{i=0}^{m} \sum_{j=0}^{n} \alpha_{i} \beta_{j} x^{i+j}=\sum_{i=0}^{m} \sum_{j=0}^{n} a_{i} b_{j} u^{-1} v^{-1} x^{i+j}=$ $\left(\sum_{i=0}^{m} \sum_{j=0}^{n} a_{i} b_{j} x^{i+j}\right)(u v)^{-1} \in N_{*}(E)[x]$, so we get $\sum_{i=0}^{m} \sum_{j=0}^{n} a_{i} b_{j} x^{i+j} \in$ $N_{*}(R)[x]$. Since $R$ is APR, $a_{i} b_{j} \in N_{*}(R)$ for all $i, j$ and so $\alpha_{i} \beta_{j}=a_{i} u^{-1} b_{j} v^{-1}=$ $a_{i} b_{j} u^{-1} v^{-1} \in N_{*}(E)$. Thus $E$ is APR. The converse is obtained by Proposition $1.9(1)$.

The ring of Laurent polynomials in $x$, coefficients in a ring $R$, consists of all formal sums $\sum_{i=k}^{n} m_{i} x^{i}$ with obvious addition and multiplication, where $m_{i} \in R$ and $k, n$ are (possibly negative) integers; written by $R\left[x ; x^{-1}\right]$.

Corollary 2.10. A ring $R$ is $A P R$ if and only if $R[x]$ is $A P R$ if and only if $R\left[x ; x^{-1}\right]$ is $A P R$.

Proof. Let $M=\left\{1, x, x^{2}, \ldots\right\}$. Then $M$ is a multiplicative monoid consisting of central regular elements in $R[x]$. Note that $R\left[x ; x^{-1}\right]=M^{-1} R[x]$. So the proof is obtained from Theorem 2.1 and Proposition 2.9.

Recall that $\operatorname{Mat}_{n}(A)(n \geq 2)$ cannot be nil-Armendariz over any ring $A$. Indeed, considering polynomials $f(x)=e_{11}-e_{12} x, g(x)=\left(e_{21}+e_{22}\right)+\left(e_{11}+\right.$ $\left.e_{12}\right) x$ in $\operatorname{Mat}_{n}(A)[x]$, then $f(x) g(x)=0$ but $e_{11}\left(e_{11}+e_{12}\right)=e_{11}+e_{12} \notin$ $N\left(\operatorname{Mat}_{n}(A)\right)$, entailing that $\operatorname{Mat}_{n}(A)$ is not nil-Armendariz. So Theorem 1.4(1) implies that $\operatorname{Mat}_{n}(A)$ is not APR. But we can find some kinds of APR subrings of $\operatorname{Mat}_{n}(A)$ in the following. 
Theorem 2.11. Given a ring $R$ the following conditions are equivalent:

(1) $R$ is $A P R$;

(2) $U_{n}(R)$ is $A P R$;

(3) $D_{n}(R)$ is $A P R$.

Proof. The proof is obtained from Lemma 1.1(4), Corollary 1.10, and the fact that a ring $A$ is APR if and only if $A / N_{*}(A)$ is Armendariz. In fact we get $N_{*}\left(U_{n}(A)\right)=\left\{m=\left(m_{i j}\right) \in U_{n}(A) \mid m_{i i} \in N_{*}(A)\right\}$ and $N_{*}\left(D_{n}(A)\right)=\{m=$ $\left.\left(m_{i j}\right) \in D_{n}(A) \mid m_{i i} \in N_{*}(A)\right\}$, so $U_{n}(R) / N_{*}\left(U_{n}(R)\right)$ is the direct product of $n$-copies of $R / N_{*}(R)$ and $\frac{D_{n}(R)}{N_{*}\left(D_{n}(R)\right)} \cong \frac{R}{N_{*}(R)}$.

Theorem 2.11 also shows that an APR ring which is not Armendariz, can always be constructed from any Armendariz ring.

The same idea as in the proof of Theorem 2.11 can be used to prove the following. As in [29, Definition 1.3], for a commutative ring $R$ with an endomorphism $\sigma$ and $R$-module $M$, we define the skewtrivial extension of $R$ by $M$ and $\sigma$ to be the ring $R \oplus M$ with multiplication $\left(r_{1}, m_{1}\right)\left(r_{2}, m_{2}\right)=\left(r_{1} r_{2}, \sigma\left(r_{1}\right) m_{2}+\right.$ $\left.r_{2} m_{1}\right)$ where $r_{i} \in R$ and $m_{i} \in M$. $(r, m)$ can be expressed by $\left(\begin{array}{cc}r & m \\ 0 & r\end{array}\right)$, and the product can be expressed by $\left(r_{1}, m_{1}\right)\left(r_{2}, m_{2}\right)=\left(\begin{array}{cc}r_{1} r_{2} & \sigma\left(r_{1}\right) m_{2}+r_{2} m_{1} \\ 0 & r_{1} r_{2}\end{array}\right)$.

Proposition 2.12. (1) Let $R_{1}, R_{2}$ be rings and $R_{1} M_{R_{2}}$ an $\left(R_{1}, R_{2}\right)$-bimodule. Then $\left(\begin{array}{cc}R_{1} & M \\ 0 & R_{2}\end{array}\right)$ is $A P R$ if and only if both $R_{1}$ and $R_{2}$ are $A P R$.

(2) Let $R$ be a commutative ring with an endomorphism $\sigma$ and $R$-module $M$. Then $R$ is APR if and only if so is the skewtrivial extension of $R$ by $M$ and $\sigma$.

Let $R$ be an algebra (with or without identity) over a commutative ring $S$. Due to Dorroh [11], the Dorroh extension of $R$ by $S$ is the Abelian group $R \oplus S$ with multiplication given by $\left(r_{1}, s_{1}\right)\left(r_{2}, s_{2}\right)=\left(r_{1} r_{2}+s_{1} r_{2}+s_{2} r_{1}, s_{1} s_{2}\right)$ for $r_{i} \in R$ and $s_{i} \in S$.

Theorem 2.13. Let $R$ be an algebra over a commutative domain $S$ where $R$ is assumed to have the identity.

(1) $R$ is APR if and only if so is the Dorroh extension $D$ of $R$ by $S$.

(2) $R$ is Armendariz if and only if so is the Dorroh extension $D$ of $R$ by $S$.

Proof. Note that $s \in S$ is identified with $s 1 \in R$, so $R=\{r+s \mid(r, s) \in D\}$. This yields $N_{*}(D)=N_{*}(R) \oplus 0$ through a simple computation.

(1) Let $R$ be APR and suppose that $f(x) g(x) \in N_{*}(D)[x]$ for $f(x)=$ $\sum_{i=0}^{m} a_{i} x^{i}, g(x)=\sum_{j=0}^{n} b_{j} x^{j} \in D[x]$. Write $a_{i}=\left(\alpha_{i}, s_{i}\right), b_{j}=\left(\beta_{j}, t_{j}\right)$ for all $i, j$. Assume that there exist $i, j$ such that $s_{i} \neq 0$ and $t_{j} \neq 0$. Say that $i_{0}$ and $j_{0}$ are the smallest such integers. Then the coefficient of $x^{i_{0}+j_{0}}$ of $f(x) g(x)$ is $\left(p, s_{0} t_{0}\right)$ for some $p \in R$, a contradiction since $\left(p, s_{0} t_{0}\right) \notin N_{*}(D)$. Thus $s_{i}=0$ for all $i$ or $t_{j}=0$ for all $j$. Write $f(x)=f_{1}(x)+f_{2}(x)$ and $g(x)=g_{1}(x)+g_{2}(x)$ with

$$
f_{1}(x)=\sum_{i=0}^{m}\left(\alpha_{i}, 0\right) x^{i}, f_{2}(x)=\sum_{i=0}^{m}\left(0, s_{i}\right) x^{i}
$$




$$
g_{1}(x)=\sum_{j=0}^{n}\left(\beta_{j}, 0\right) x^{j}, g_{2}(x)=\sum_{j=0}^{n}\left(0, t_{j}\right) x^{j} .
$$

Suppose that $s_{i}=0$ for all $i$, i.e., $f_{2}(x)=0$. Then we have

$$
\begin{aligned}
f(x) g(x) & =f_{1}(x) g_{1}(x)+f_{1}(x) g_{2}(x) \\
& =\sum_{i+j=0}^{m+n}\left(\alpha_{i} \beta_{j}, 0\right) x^{i+j}+\sum_{i+j=0}^{m+n}\left(\alpha_{i} t_{j}, 0\right) x^{i+j} \\
& =\sum_{i+j=0}^{m+n}\left(\alpha_{i}\left(\beta_{j}+t_{j}\right), 0\right) x^{i+j} \in\left(N_{*}(R) \oplus 0\right)[x] .
\end{aligned}
$$

Since $R$ is APR, $\alpha_{i}\left(\beta_{j}+t_{j}\right) \in N_{*}(R)$ for all $i, j$. This yields $a_{i} b_{j}=\left(\alpha_{i}, 0\right)\left(\beta_{j}, t_{j}\right)$ $\in N_{*}(D)$ for all $i, j$.

The computation for the case of $g_{2}(x)=0$ is similar. Thus $D$ is also APR.

Next since $N_{*}(R)=N_{*}(D)$ through the inclusion map $r \mapsto(r, 0)$ for $r \in R$, Proposition 1.9(1) completes the proof.

(2) The proof is similarly obtained by letting $f(x) g(x)=0$ in place of $f(x) g(x) \in N_{*}(D)[x]$.

Next we examine the case of $R$ being assumed to do not have the identity. In this case the Dorroh extension $D$, in Theorem 2.13, means a ring extension to attach an identity to $R$; hence we cannot use the fact that $s \in S$ is identified with $s 1 \in R$ and $R=\{r+s \mid(r, s) \in D\}$.

Proposition 2.14. Let $R$ be an algebra over a commutative domain $S$ where $R$ is assumed to do not have the identity. If the Dorroh extension $D$ of $R$ by $S$ is $A P R$, then so is $R$.

Proof. We will show that $N_{*}(R) \oplus 0=N_{*}(D)$. This yields that if $D$ is APR, then $R$ is APR by Proposition 1.9(1) since $N_{*}(R)=N_{*}(D)$ through the inclusion map $r \mapsto(r, 0)$ for $r \in R$. Every nilpotent element in $D$ is clearly of the form $(a, 0)$, so we get $N_{*}(R) \oplus 0 \supseteq N_{*}(D)$. To show the converse inclusion, let $z_{1}=a \in N_{*}(R)$ and $v_{1}=(a, 0) \in D$. Consider a sequence

$$
w_{1}=v_{1} d_{1} v_{1}, w_{2}=w_{1} d_{2} w_{1}, \ldots, w_{k+1}=w_{k} d_{k} w_{k}, \ldots
$$

for $k=1,2, \ldots$, where every $d_{k}(j=1,2, \ldots)$ is taken arbitrarily in $D$. Say $d_{k}=\left(r_{k}, s_{k}\right)$. Then

$$
w_{1}=\left(a r_{1} a+a s_{1} a, 0\right)=\left(a b_{1}, 0\right)=\left(e_{1} a, 0\right),
$$

where $b_{1}=r_{1} a+s_{1} a, e_{1}=a r_{1}+a s_{1}$;

$$
w_{2}=\left(a b_{1} r_{2} e_{1} a+a b_{1} s_{2} e_{1} a, 0\right)=\left(a\left(b_{1} r_{2} e_{1}+b_{1} s_{2} e_{1}\right) a, 0\right),
$$

write $z_{2}=a\left(b_{1} r_{2} e_{1}+b_{1} s_{2} e_{1}\right) a \in a R a=z_{1} R z_{1}$;

$$
w_{3}=\left(z_{2} r_{3} z_{2}+z_{2} a_{3} z_{2}, 0\right)=\left(z_{2} b_{2}, 0\right)=\left(e_{2} z_{2}, 0\right),
$$


where $b_{2}=r_{3} z_{2}+a_{3} z_{2}, e_{2}=z_{2} r_{3}+z_{2} a_{3}$

$$
w_{4}=\left(z_{2} b_{2} r_{4} e_{2} z_{2}+z_{2} b_{2} s_{4} e_{2} z_{2}, 0\right)=\left(z_{2}\left(b_{2} r_{4} e_{2}+b_{2} s_{4} e_{2}\right) z_{2}, 0\right),
$$

write $z_{3}=z_{2}\left(b_{2} r_{4} e_{2}+b_{2} s_{4} e_{2}\right) z_{2} \in z_{2} R z_{2}$.

Proceeding in this manner, we obtain a sequence in $R$

$$
z_{1}, z_{2}, \ldots, z_{m}, z_{m+1}, \ldots \text { with } z_{m+1} \in z_{m} R z_{m} \text { and } w_{2 m}=\left(z_{m+1}, 0\right) \text {. }
$$

But $z_{1} \in N_{*}(R)$, so $z_{l}=0$ for some $l \geq 1$. This yields $w_{2(l-1)}=0$, entailing $v_{1}=(a, 0) \in N_{*}(D)$. Thus $N_{*}(R) \oplus 0 \subseteq N_{*}(D)$, so we now have $N_{*}(R) \oplus 0=$ $N_{*}(D)$.

We actually do not know whether the converse of Proposition 2.14 is true.

Question. Let $R$ be an algebra over a commutative domain $S$ where $R$ is assumed to do not have the identity. Is $D$ APR when $R$ is APR?

\section{References}

[1] D. D. Anderson and V. Camillo, Armendariz rings and Gaussian rings, Comm. Algebra 26 (1998), no. 7, 2265-2272.

[2] R. Antoine, Nilpotent elements and Armendariz rings, J. Algebra 319 (2008), no. 8, 3128-3140.

[3] Examples of Armendariz rings, Comm. Algebra 38 (2010), no. 11, 4130-4143.

[4] E. P. Armendariz, A note on extensions of Baer and P.P.-rings, J. Austral. Math. Soc. 18 (1974), 470-473.

[5] E. P. Armendariz, H. K. Koo, and J. K. Park, Isomorphic Ore extensions, Comm. Algebra 15 (1987), no. 12, 2633-2652.

[6] G. M. Bergman, Coproducts and some universal ring constructions, Trans. Amer. Math. Soc. 200 (1974), 33-88.

[7] _ Modules over coproducts of rings, Trans. Amer. Math. Soc. 200 (1974), 1-32.

[8] G. F. Birkenmeier, H. E. Heatherly, and E. K. Lee, Completely prime ideals and associated radicals, Ring theory (Granville, OH, 1992), 102-129, World Sci. Publ., River Edge, NJ, 1993.

[9] G. F. Birkenmeier, J. Y. Kim, and J. K. Park, Regularity conditions and the simplicity of prime factor rings, J. Pure Appl. Algebra 115 (1997), no. 3, 213-230.

[10] Y. U. Cho, N. K. Kim, M. H. Kwon, and Y. Lee, Classical quotient rings and ordinary extensions of 2-primal rings, Algebra Colloq. 13 (2006), no. 3, 513-523.

[11] J. L. Dorroh, Concerning adjunctions to algebras, Bull. Amer. Math. Soc. 38 (1932), no. $2,85-88$.

[12] K. E. Eldridge, Orders for finite noncommutative rings with unity, Amer. Math. Monthly 73 (1966), 512-514.

[13] K. R. Goodearl, Von Neumann Regular Rings, Pitman, London, 1979.

[14] K. R. Goodearl and R. B. Warfield, Jr., An Introduction to Noncommutative Noetherian Rings, Cambridge University Press, 1989.

[15] C. Huh, H. K. Kim, and Y. Lee, Questions on 2-primal rings, Comm. Algebra 26 (1998), no. 2, 595-600.

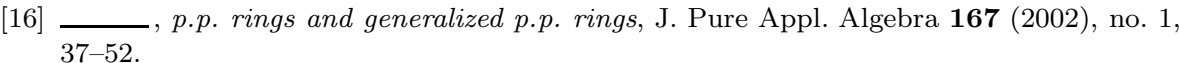

[17] C. Huh, H. K. Kim, D. S. Lee, and Y. Lee, Prime radicals of formal power series rings, Bull. Korean Math. Soc. 38 (2001), no. 4, 623-633.

[18] C. Huh, Y. Lee, and A. Smoktunowicz, Armendariz rings and semicommutative rings, Comm. Algebra 30 (2002), no. 2, 751-761. 
[19] S. U. Hwang, Y. C. Jeon, and Y. Lee, Structure and topological conditions of NI rings, J. Algebra 302 (2006), no. 1, 186-199.

[20] S. U. Hwang, Y. Lee, and K. S. Park, On strongly 2-primal rings, Honam Mathematical J. 29 (2007), no. 4, 555-567.

[21] Y. C. Jeon, H. K. Kim, Y. Lee, and J. S. Yoon, On weak Armendariz rings, Bull. Korean Math. Soc. 46 (2009), no. 1, 135-146.

[22] D. W. Jung, N. K. Kim, Y. Lee, and S. P. Yang, Nil-Armendariz rings and upper nilradicals, Int. J. Alg. Comp. (to appear).

[23] N. K. Kim, K. H. Lee, and Y. Lee, Power series rings satisfying a zero divisor property, Comm. Algebra 34 (2006), no. 6, 2205-2218.

[24] N. K. Kim and Y. Lee, Armendariz rings and reduced rings, J. Algebra 223 (2000), no. $2,477-488$

[25] N. K. Kim, Y. Lee, and S. J. Ryu, An ascending chain condition on Wedderburn radicals, Comm. Algebra 34 (2006), no. 1, 37-50.

[26] T. Y. Lam, A First Course in Noncommutative Rings, Springer-Verlag, New York, 1991.

[27] J. Lambek, Lectures on Rings and Modules, Blaisdell Publishing Company, Waltham, 1966.

[28] G. Marks, On 2-primal Ore extensions, Comm. Algebra 29 (2001), no. 5, 2113-2123.

[29] M. B. Rege and S. Chhawchharia, Armendariz rings, Proc. Japan Acad. Ser. A Math. Sci. 73 (1997), no. 1, 14-17.

JunChEOL HAN

Department of Mathematics Education

Pusan National University

PUSAn 609-735, Korea

E-mail address: jchan@pusan.ac.kr

HONG KeE KIM

Department of Mathematics and RINS

Gyeongsang National University

JINJU 660-701, KOREA

E-mail address: hkkim@gsnu.ac.kr

YANG LEE

Department of Mathematics Education

Pusan National University

Pusan 609-735, Korea

E-mail address: ylee@pusan.ac.kr 